\title{
Breaching the Axial Limits in Ln(III) Single-Ion Magnets Using Ex- ternal Electric Field
}

\author{
Arup Sarkar, and Gopalan Rajaraman* \\ Department of Chemistry, Indian Institute of Technology Bombay, Powai, Mumbai- 400076. \\ E-mail: rajaraman@chem.iitb.ac.in
}

KEYWORDS: Single-Ion Magnets $\bullet$ External Electric FIeld $\bullet$ CASSCF $\bullet$ Magnetic Anisotropy.

\begin{abstract}
Single-Molecule Magnets have potential applications in several nano-technology applications including in high-dense information storage devices and realization of this potential application lies in enhancing the barrier height for magnetization reversal $\left(U_{\text {eff }}\right)$. Recent literature examples suggest that the maximum values that one can obtain using a ligand field are already accomplished. Here we have explored using a combination of DFT and ab initio CASSCF calculations, the way to enhance the barrier height using an oriented external electric field for top three Single-ion Magnets $\left(\left[\mathrm{Dy}(\mathrm{Py})_{5}\left(\mathrm{O}^{t} \mathrm{Bu}\right)_{2}\right]^{+}(\mathbf{1}) \text { and }\left[\mathrm{Er}\left\{\mathrm{N}(\mathrm{SiMe})_{3}\right)_{2}\right\}_{3} \mathrm{Cl}\right]^{-}(\mathbf{2})$ and $\left.\left[\mathrm{Dy}\left(\mathrm{Cp}^{\mathrm{Me} 3}\right) \mathrm{Cl}\right](\mathrm{3})\right)$. For the first time our study reveals that, for apt molecules, if appropriate direction and value of electric fields are chosen, the barrier height could be enhanced twice that of the limit set by the ligand field. This novel non-chemical-fine tuning approach to modulate the magnetic anisotropy is expected to yield new generation SIMs.
\end{abstract}

There is a great interest in the area of single-molecule magnets (SMMs) as they are reported to have potential applications in information storage devices, cryogenic refrigeration, quantum computing and spintronics devices to name a few. ${ }^{1}$ SMMs containing Lanthanide(III) ions are gaining interest in recent years as they possess huge barrier height for magnetization reversal $\left(\mathrm{U}_{\text {eff }}\right)$ and at the same time possess record high blocking temperatures $\left(\mathrm{T}_{\mathrm{B}}\right)$. While there are various classes of molecules exhibit blocking temperatures in the range of $4-15 \mathrm{~K},{ }^{2}$ higher blocking temperatures are found for organometallic Dy(III) single-ion magnets (SIMs) containing substituted cyclopentadienyl ligands $\left(\mathrm{T}_{\mathrm{B}}\right.$ in the range of $48 \mathrm{~K}$ to $\left.80 \mathrm{~K}\right) .^{3}$

It is well-known that the shape of the electron density of the ground state $m_{J}$ levels of the Lanthanide ion is critical in dictating their magnetic properties. The Ln(III) ions are classified as those possessing oblate density require strong axial with no/weak equatorial ligation and those with prolate density demands strong equatorial ligand with weak/no axial ligation. Synthetic chemists have been utilizing these ideas to develop novel molecules with attractive $\mathrm{U}_{\text {eff }}$ and $\mathrm{T}_{\mathrm{B}}$ values. ${ }^{4}$ While most of the molecules possessing very high-blocking temperature also possess substantial $U_{\text {eff }}$ values, often the $T_{B}$ is only a fraction of the $\mathrm{U}_{\text {eff }}$ values reported. While establishing the relationship between the $U_{\text {eff }}$ and $T_{B}$ and mechanism beyond the singleion relaxation has gained attention, ${ }^{5}$ it is also equally important to realize large $\mathrm{U}_{\text {eff }}$ values to move forward.

To obtain a larger $U_{\text {eff }}$ value for lanthanide complexes, various chemical fine-tuning methods such as (i)designer ligands that control the ligand field around the $\mathrm{Ln}$ (III) ion in an anticipated fashion, ${ }^{6}$ (ii) maintaining symmetry around the metal centre, ${ }^{2,7}$ (iii) incorporating diamagnetic elements in the cluster aggregation to enhance the axiality ${ }^{8}$ or (iv) incorporate transition metal or radicals to induce exchange interaction as a way to suppress tunneling have been explored. ${ }^{4,9}$ With numerous Dy(III) mononuclear complexes reported in the literature, it has been stated that the axial limit that controls the overall $U_{\text {eff }}$ value has been reached. ${ }^{2 a}$ While increasing $T_{B}$ value being the focus at present, other avenues to enhance the $U_{\text {eff }}$ values have not been explored. As chemical fine-tuning of the ligand field has already reached its potential, we aim to search an alternative route to enhance the $\mathrm{U}_{\text {eff }}$ values in Ln(III) SIMs. In this context, using various computational tools, here we set out to explore the role of an applied electric field in the magnetization reversal of Ln(III) SIMs. Recent examples in this area where electric field has been utilized to modulate the magnetic properties offers strong motivation for this work. ${ }^{10}$ To enumerate the effected of an oriented external electric field (OEEF) on Lanthanide SIMs, we have chosen three examples $\left[\mathrm{Dy}(\mathrm{Py})_{5}\left(\mathrm{O}^{\mathrm{t} B u}\right)_{2}\right]\left[\mathrm{BPh}_{4}\right](\mathbf{1})^{2 \mathrm{a}}$ $\mathrm{Li}(\mathrm{THF})_{4}\left[\mathrm{Er}\left\{\mathrm{N}\left(\mathrm{SiMe}_{3}\right)_{2}\right\}_{3} \mathrm{Cl}\right]^{11}$ (2) and $\left[\mathrm{Dy}\left(\mathrm{Cp}^{\mathrm{Me} 3}\right) \mathrm{Cl}\right]^{3 \mathrm{a}}$ (3) complexes. All three complexes are characterized well and among the best-known SIMs in their family. Particularly complex 1 found to exhibit a $U_{\text {eff }}$ value of $1815 \mathrm{~K}$ with a blocking temperature of $14 \mathrm{~K}$ while complex 2 found to have $\mathrm{U}_{\text {eff }}$ value of $63 \mathrm{~K}$ with a $\mathrm{T}_{\mathrm{B}}$ of $3 \mathrm{~K}$. Complex 3 , on the other hand, do not exhibit any out-of-phase signals and hence is not a Single-ion magnet. $^{3 \mathrm{c}}$

Computing the magnetic anisotropy of the Ln(III) SIMs in the presence of electric field has not been attempted, and there are multiple challenges present to account for such effects. The application of oriented electric fields is expected to distort the geometry and capturing this effect is crucial in understanding the magnetic anisotropy. As Ln(III) SIMs are known to be extremely sensitive to small structural changes, static OEEF on Xray structure unlikely to reveal the real scenario. As structure optimization with the ab initio CASSCF calculations is not practical at present, here we have chosen a combination of 
methodology wherein DFT calculations in the presence of electric field were performed to obtain reasonable structures. These structures were then subject to ab initio CASSCF/RASSISO/SINGLE_ANISO calculations in the presence of same electric field to capture both the structural distortion and also the electric field effect on the magnetic anisotropy (see computational details for more information). Ab initio calculations were performed on the crystal structures of complexes (or models derived from the X-ray structure) of $\mathbf{1 ~ - 3}$ in the absence of any external perturbation (see Table S1-S3 in ESI). Complex 1 and $\mathbf{2}$ are well-known examples, exhibiting strong axiality in the estimated $\mathrm{g}_{\mathrm{z}}$ values with the computed barrier height of $1183 \mathrm{~cm}^{-}$ ${ }^{1}$ and $181 \mathrm{~cm}^{-1}$, respectively (relaxation via $4^{\text {th }}$ excited Kramers doublet). ${ }^{2 \mathrm{a}, 12}$.

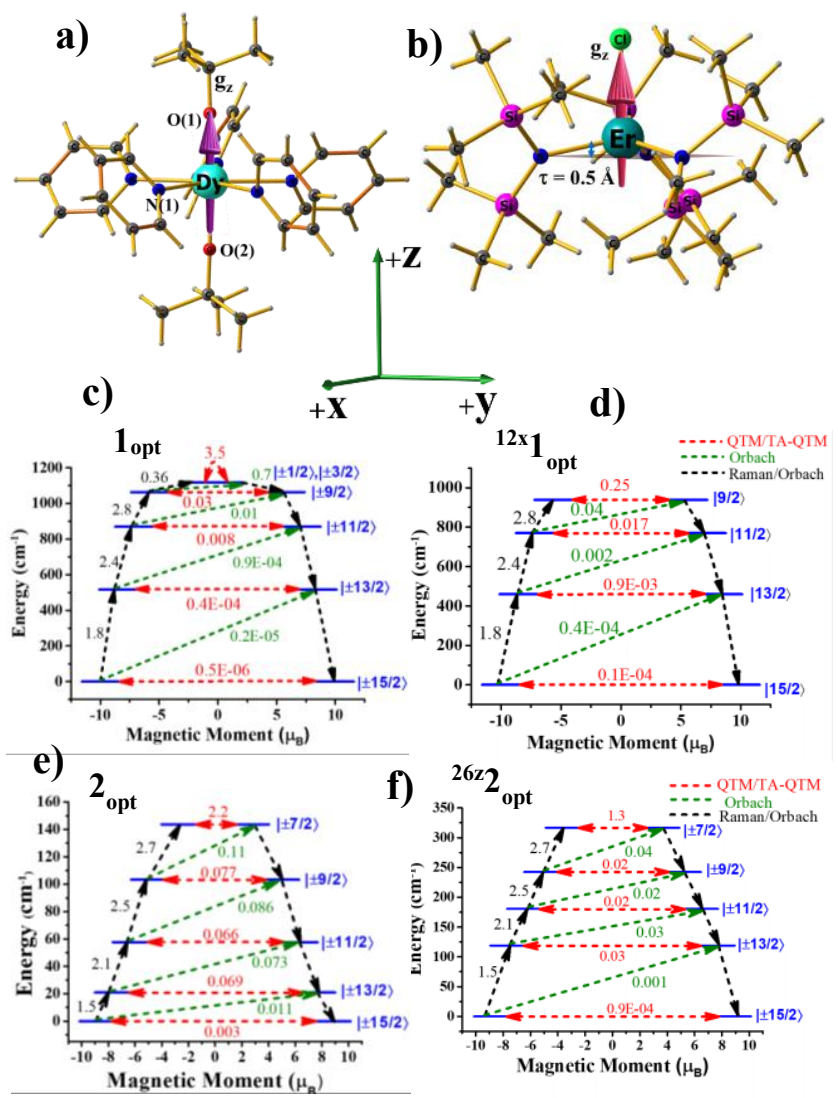

Figure 1. a) Structures of $\mathbf{1}_{\text {opt }}$ and b) $\mathbf{2}_{\text {opt }}$ along with computed $\mathrm{g}_{\mathrm{z}}$-axis. Colour codes: Dy: cyan, Er: dark cyan, N: blue, $\mathrm{Cl}$ : green, $\mathrm{Si}$ : pink, $\mathrm{C}$ : grey and $\mathrm{H}$ : light grey. c) $\mathrm{Ab}$ initio blocking barrier and relaxation mechanism of $\left.\mathbf{1}_{\text {opt }}, d\right){ }^{12 \times} \mathbf{1}_{\text {opt }}$, e) $\mathbf{2}_{\text {opt }}$ and f) ${ }^{26 z} \mathbf{2}_{\text {opt }}$.

As geometries of $\mathbf{1}$ and $\mathbf{2}$ are relaxed in the presence of an electric field, it is imperative to understand how the optimized geometry in the gas phase fair to the X-ray structure. The optimized geometry of complexes (1 $\mathbf{1}_{\mathrm{opt}}$ and $\mathbf{2}_{\mathrm{opt}}$ ) reveal elongation of all the bonds within the molecule as intermolecular interactions in the crystal lattice are removed. The axial $\mathrm{Dy}-\mathrm{O}(1)$ bond length increases from $2.110 \AA$ in the X-ray structure to $2.142 \AA$ in $\mathbf{1}_{\text {opt, }}$ and the average equatorial Dy-N bond length also increases $\sim 0.05 \AA$ in $\mathbf{1}_{\text {opt }}$ geometry (see Table 1). Similar elongation has been witnessed in Er-N/Cl bond lengths in complex 2 . The CASSCF calculations on $\mathbf{1}_{\text {opt }}$ and $\mathbf{2}_{\text {opt }}$ yield $U_{\text {cal }}$ value of $1118 \mathrm{~cm}^{-1}$ and $144 \mathrm{~cm}^{-1}$, respectively, assuming relaxation via $4^{\text {th }}$ excited state (See Fig. 1) and these values are marginally smaller than the X-ray geometry due to relatively weaker axial ligand field (LF) in optimized geometries (see Table S4-S5).

In the next step, we have attempted to optimize the geometry in the presence of oriented external electric field (OEEF) starting from $0.004 \mathrm{au}$ (atomic unit and equivalent to $0.2 \mathrm{~V} / \AA$ ) $)^{10 \mathrm{c}, 13}$ The electric field applied here varies from 0.004 au to 0.026 au and lies within the limit of ionization energies, bond dissociation energies and is accessible for most of the STM tips. ${ }^{13-14}$ While the electric field induced spectroscopic techniques uses smaller field, organic reactions performed using OEFF are comparable to the electric field utilized here. ${ }^{13-14}$

Applying the electric field along the $+\mathrm{z}$-axis in $\mathbf{1}_{\text {opt }}$ (See Fig. 1a and Fig. S1 in ESI), elongates the Dy-O(1) bond and at the same time shortens the $\mathrm{Dy}-\mathrm{O}(2)$ bond and therefore breaks the pseudo $\mathrm{D}_{5 \mathrm{~h}}$ symmetry of the molecule. We have performed $\mathrm{ab}$ initio CASSCF calculations on this optimized geometry ${ }^{\mathbf{4}} \mathbf{1}_{\mathbf{o p t}}$ (here superscript denotes the amount of OEEF applied x $10^{-3}$ au along $+\mathrm{z}$ direction) in the presence of electric field (EF) wherein a reduction in the barrier height was witnessed. This is due to the fact that Dy-O(1) elongation cause the weakening of the axial LF and hence reduces the axial anisotropy for the oblate Dy(III) ion. Although there is a simultaneous shortening of Dy$\mathrm{O}(2)$ is noticed, ${ }^{\mathbf{4}} \mathbf{1}_{\mathbf{o p t}}$ geometry reveal that elongation is greater than the shortening (see Fig. S1) and hence this is not fully symmetric leading to a smaller $U_{\text {cal }}$ value of $1108 \mathrm{~cm}^{-1}$ for ${ }^{\mathbf{4}} \mathbf{1}_{\text {opt. }}$. In the next step, we increase the OEEF value in a stepwise manner to 0.012 au and see clearly that increase in the electric field increase the Dy-O(1) bond further and at the same time shortens the $\mathrm{Dy}-\mathrm{O}(2)$ bond albeit asymmetrically. This led to a further reduction in the barrier height with a value of $1040 \mathrm{~cm}^{-1}$ noted for ${ }^{122} 1_{\text {opt }}$ structure (see Table S6 and S9-S11 in ESI). This reduction in barrier height can be rationalized by analyzing LoProp charges at the spin-free ground state. By increasing OEEF, the LoProp charge on $\mathrm{O}(1)$ gradually decreases while it increases in $\mathrm{O}(2)$ (see Table S8 and S16). Perceiving this effect, we switched the OEEF direction along the $\mathrm{x} / \mathrm{y}$ direction of complex $\mathbf{1}_{\text {opt }}$ (see Fig. S1 in ESI). The optimized structure of ${ }^{\mathbf{4}} \mathbf{1}_{\text {opt }}$ (here superscript denoted the amount of OEEF applied $\times 10^{-3}$ au along $+\mathrm{x}$ direction). Here $\mathrm{Dy}-\mathrm{N}(1)$ bond length found to increase sharply from $2.62 \AA$ to $2.80 \AA$ vis a vis $\mathbf{1}_{\text {opt }}$ vs ${ }^{{ }^{12 x}} \mathbf{1}_{\text {opt }}$ (see Table 1) geometry and at the same time two of the Dy-N (along the $-\mathrm{x}$ direction) found to shorten asymmetrically. Also, the effect of applying OEEF along Dy-N(1) direction can be seen in a substantial decrease in the LoProp charge of N(1) atom while the charges on the oxygen atoms remain unaltered (see Table S8 in ESI). As three Dy-N bonds are significantly elongated at ${ }^{12 x} 1_{\text {opt }}$ geometry, one could expect a large barrier height, however, ab initio calculations reveal a contrary with a barrier height diminishing with an increase in OEEF value yielding a $\mathrm{U}_{\text {cal }}$ value of $939 \mathrm{~cm}^{-1}$ for ${ }^{\mathbf{1 2 x}} \mathbf{1}_{\text {opt }}$ which relaxes via $3^{\text {rd }}$ excited KDs. (see Table S6 and S12-S14 in ESI). This is due to alteration of Dy-N distances that are accompanied by a variation in the O-Dy-O angle, which is reduced to $157^{\circ}$ in ${ }^{\mathbf{1 2 x}} \mathbf{1}_{\text {opt }}$ from $178^{\circ}$ in $\mathbf{1}_{\text {opt }}$ geometry (see Table 1). Thus, the application of the electric field along the perpendicular or $\mathrm{g}_{\mathrm{x}}$-direction worsens the barrier height for complex 1. To prove that the reduction is solely due to the O-Dy-O bending, we have performed one additional set of calculation on ${ }^{\mathbf{1 2 x}} \mathbf{1}_{\text {opt }}$ geometry wherein the O-Dy$\mathrm{O}$ angle is fictitiously set at $178^{\circ}$ and this structure yield a barrier height of $1162 \mathrm{~cm}^{-1}$ (See Fig. S2 and Table S15 in ESI). This value estimated is $\sim 50 \mathrm{~cm}^{-1}$ higher compared to optimized geometry offering a possibility, however small, to enhance $U_{c a l}$ 
value in 1 using an applied electric field. Furthermore, increasing the OEEF at 0.016 au results in dissociation of the Dy-N bond, which sets the electric field limit at $\mathrm{x} / \mathrm{y}$ direction of the molecule.

To further understand how the structure alteration occurs due to OEEF, it is important to understand the nature of dipoles and their behavior in the applied electric field conditions. Application of OEEF expected to polarize a non-polar bond and enhance the ionic character of a polar bond. ${ }^{13}$ For a Ln-L bond, the application of OEEF will stretch it further if the dipolar field creates opposite dipole with respect to the Ln-L dipole and will help to shorten it, if the dipolar field is in the same direction as the Ln- L dipole (see Fig. 2b). Therefore, the molecule has to be chosen in such a way that an increase in the Ln-L bond length will enhance the magnetic anisotropy and will subsequently increase barrier height ( $\left.U_{\text {eff }}\right)$.

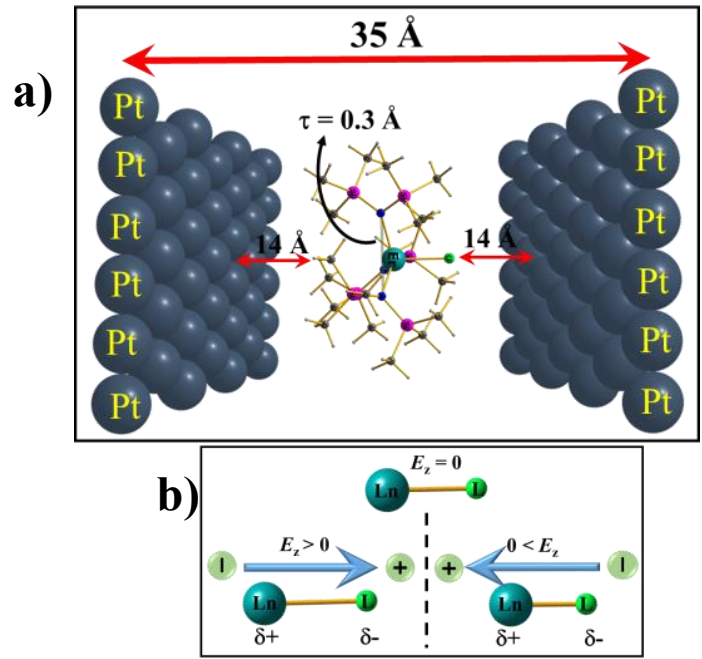

Figure 2. a) Arrangement of applying external electric field by putting point charges on two opposite $\mathrm{Pt}$ (111) layers which are $35 \AA$ apart and the molecule is at the centre during ab initio calculations (the distance between molecule to Pt layers is not be scale). b) Result of applying OEEF on a polar Ln-L bond axis.

Table 1: Selected structural parameters of complexes $\mathbf{1}$ and $\mathbf{2}$ in the presence of an electric field. Bond lengths, $\tau$ in $\AA$ unit, angles are in $\left(^{\circ}\right)$ and $U_{\text {cal }}$ values in $\mathrm{cm}^{-1}$.

\begin{tabular}{|c|c|c|c|c|c|c|c|}
\hline & $1_{\text {opt }}$ & ${ }^{4 z} \boldsymbol{I}_{\text {opt }}$ & ${ }^{8 z} \boldsymbol{1}_{\text {opt }}$ & ${ }^{12 z} \boldsymbol{I}_{\text {opt }}$ & ${ }^{4 x} 1_{\text {opt }}$ & ${ }^{8 x} 1_{\text {opt }}$ & ${ }^{12 x} 1_{\text {opt }}$ \\
\hline$D y-O 1$ & 2.141 & 2.170 & 2.203 & 2.244 & 2.138 & 2.138 & 2.139 \\
\hline Dy-O2 & 2.142 & 2.118 & 2.098 & 2.081 & 2.140 & 2.139 & 2.139 \\
\hline$D y-N 1$ & 2.616 & 2.604 & 2.605 & 2.604 & 2.649 & 2.710 & 2.798 \\
\hline$D y-N 2$ & 2.610 & 2.616 & 2.616 & 2.619 & 2.604 & 2.574 & 2.554 \\
\hline$D y-N 3$ & 2.618 & 2.626 & 2.625 & 2.628 & 2.604 & 2.582 & 2.559 \\
\hline Dy-N4 & 2.618 & 2.606 & 2.608 & 2.607 & 2.615 & 2.642 & 2.674 \\
\hline Dy-N5 & 2.612 & 2.629 & 2.627 & 2.626 & 2.617 & 2.630 & 2.649 \\
\hline$O 1-D y-O 2$ & 178.3 & 178.5 & 178.3 & 178.0 & 171.2 & 164.6 & 157.2 \\
\hline \multirow[t]{2}{*}{$U_{c a l}$} & 1118 & 1108 & 1083 & 1040 & 1111 & 1070 & 939 \\
\hline & $2_{\text {opt }}$ & ${ }^{4 z} 2_{o p t}$ & ${ }^{8 \mathrm{z}} \mathbf{2}_{\mathrm{opt}}$ & ${ }^{12} 2_{\text {opt }}$ & ${ }^{16} z_{\text {opt }}$ & ${ }^{20 z} 1_{\text {opt }}$ & ${ }^{26 z} 1_{\text {opt }}$ \\
\hline $\mathrm{Er}-\mathrm{Cl}$ & 2.586 & 2.614 & 2.647 & 2.686 & 2.736 & 2.803 & 3.042 \\
\hline$E r-N I$ & 2.308 & 2.304 & 2.301 & 2.298 & 2.295 & 2.293 & 2.285 \\
\hline$E r-N 2$ & 2.308 & 2.304 & 2.301 & 2.298 & 2.295 & 2.292 & 2.284 \\
\hline$E r-N 3$ & 2.309 & 2.306 & 2.303 & 2.302 & 2.300 & 2.300 & 2.296 \\
\hline$\tau$ & 0.508 & 0.488 & 0.468 & 0.446 & 0.419 & 0.385 & 0.293 \\
\hline$U_{c a l}$ & 144 & 163 & 178 & 200 & 223 & 250 & 317 \\
\hline
\end{tabular}

Applying the OEEF along an equatorial Ln-L bond in oblate ions such as $\mathrm{Dy}^{3+}$ or along an axial Ln-L bond in prolate such as $\mathrm{Er}^{3+}$ or $\mathrm{Yb}^{3+}$ thus likely to increase the $U_{\text {eff }}$ value beyond the $\mathrm{X}$-ray structure reported values. However, if the OEEF is applied along the opposite directions, this is expected to decrease the $U_{\text {eff }}$ value further.

Based on the knowledge gained, we intuitively expand the study to a prolate $\mathrm{Er}$ (III) ion using complex 2 . We have narrow down to this example for two reasons (i) to choose a well-studied prolate $\operatorname{Er}($ III) SIM with a significant barrier height (ii) to choose an $\operatorname{Er}($ III) SIM with a strong equatorial ligand and a weak axial ligand along only one direction as this would be expected to facilitate the enhancement of the $U_{\text {cal }}$ value upon application of OEEF. Upon applying the OEEF along the Er-Cl direction ( $g_{z}$ axis, see Fig. 2), with the same step size as before, the Er-Cl bond length found to increase significantly (see Fig. S3 in ESI and Table 1) reaching a maximum value of $3.04 \AA$ at 0.026 au $\mathrm{E}_{\mathrm{Z}}\left({ }^{\mathbf{2 6 z}} \mathbf{2} \mathrm{opt}\right)$. The application of OEEF beyond this value found to cleave Er-Cl bond suggesting possible ionization/decomposition limit.

Additionally, the $\left\{\mathrm{N}_{3} \mathrm{Er}\right\}$ out-of-plane pyramidal shift (parameter $\tau$ see Fig. 2 and S3 in ESI) also found to alter upon application of OEEF. As OEEF is applied along the Er-Cl bond, this bond elongates and also pushes the $\mathrm{Er}^{3+}$ ion down and hence decreases the $\tau$ value. The $\tau$ value decreases from $0.5 \AA$ at the $\mathbf{2}_{\text {opt }}$ to $0.3 \AA$ at ${ }^{26 z} \mathbf{2}_{\text {opt. }}$ If the OEEF is applied along the $-\mathrm{z}$ direction (Cl-Er direction), this tends to enhance the pyramidalization (see Fig. S3 in ESI) and thus, $\tau$ value increases to $0.62 \AA$ at ${ }^{26-z} 2_{\text {opt. }}$ Theoretical studies performed earlier on complex 2 reveal that this is an important parameter that enhances the barrier height. ${ }^{15}$

Application of OEEF along the $\mathrm{g}_{\mathrm{z}}$ in 2 (.i.e along Er-Cl bond) axis enhances the $U_{\text {cal }} 163 \mathrm{~cm}^{-1}$ at ${ }^{\mathbf{4}} \mathbf{2}_{\text {opt }}$ to a remarkable $317 \mathrm{~cm}^{-}$ ${ }^{1}$ at ${ }^{26 z} 2_{\text {opt. }}$ This estimate is one of the highest obtained for any Er(III) SIMs. ${ }^{16}$ Computed QTM (and TA-QTM) values reveal a smooth decrease of these values from 2.2 at ${ }^{\mathbf{4}} \mathbf{2}_{\text {opt }}$ to 1.3 at ${ }^{26 z} 2_{\text {opt. }}$ (see Table S17-S24 in ESI). Also, a smooth linear increase of the negative $B_{2}{ }^{0}$ parameter was observed for complex 2 under the applied electric field range along the $+\mathrm{z}$ direction (see Fig. S4 and Table S27 in ESI). If OEEF applied on the reverse direction on complex 2 , i.e., along the $-\mathrm{z}$ direction, a reverse trend was visible with a gradual decrease in $U_{\text {cal }}$ value. As expected, here the $\mathrm{Er}(\mathrm{III})-\mathrm{Cl}$ bond length decreases and a $\tau$ value were noticed upon applying an electric field in the $-\mathrm{z}$ direction. The $\mathrm{U}_{\text {cal }}$ value decreases from $131 \mathrm{~cm}^{-1}$ at ${ }^{4-z} \mathbf{2}_{\text {opt }}$ to a much smaller value of $52 \mathrm{~cm}^{-1}$ (via $3^{\text {rd }}$ excites state) at ${ }^{\mathbf{2 4 - z}} \mathbf{2}_{\text {opt }}$ structure (see Table S25-S27 at ESI). Further, the $\mathrm{U}_{\text {cal }}$ vanishes to zero at ${ }^{\mathbf{2 6 - z}} \mathbf{2}_{\text {opt }}$ with a notable ground state QTM. We have also plotted the $\beta$-electron density of the Er(III) under the applied electric field conditions, and this nicely reflects the changes observed (see Fig. S5 for plot corresponding to ${ }^{\mathbf{2 6 - z}} \mathbf{2}_{\mathbf{o p t}}$, $\boldsymbol{2}_{\text {opt and }}{ }^{26 z} 2_{\text {opt}}$ ).

After achieving such a large $U_{\text {cal }}$ value for complex 2, we extended the study further to another Dy(III) example namely $\left[\mathrm{Dy}\left(\mathrm{Cp}^{\mathrm{Me} 3}\right)_{2} \mathrm{Cl}\right]$ (complex 3) $\left(\mathrm{Cp}^{\mathrm{Me} 3}=\right.$ trimethylcyclopentadienyl) (see Figure $\mathbf{S 6}$ top) which is a model complex derived from X-ray structure of the famous precursor $\left[\mathrm{Dy}\left(\mathrm{Cp}^{\mathrm{ttt}}\right)_{2} \mathrm{Cl}\right]{ }^{3 \mathrm{a}}$ Our calculations on the optimized structure reveals a very small $\mathrm{U}_{\mathrm{cal}}$ value of $144 \mathrm{~cm}^{-1}$ relaxing via first excited state due to high QTM being operation due to the coordination of $-\mathrm{Cl}$ along the equatorial direction (see Table S28 in ESI). To quench this 
QTM, we have applied the OEEF along the Dy-Cl bond direction and this leads to weakening of $\mathrm{Dy}-\mathrm{Cl}$ bond and gradually the $U_{\text {cal }}$ value increases from $160 \mathrm{~cm}^{-1}$ at ${ }^{4 \mathbf{z}} \mathbf{3}_{\text {opt }}$ to $519 \mathrm{~cm}^{-1}$ at ${ }^{22 z} 3_{\text {opt }}$ structure (see Table $\mathbf{S 2 8}$ and Figure S7). The Dy-Cl bond length increases from $2.59 \AA$ at ${ }^{\mathbf{4} z} \mathbf{3}_{\text {opt }}$ to $2.94 \AA$ at ${ }^{\mathbf{2 2 z}} \mathbf{3}_{\text {opt. }}$. As the Dy-Cl bond increases with the electric field, a simultaneous increase of Cp-Dy-Cp angle was observed. Application of the electric field beyond 0.022 au results in rupture of the $\mathrm{Dy}-\mathrm{Cl}$ bond. Thus, the $\mathrm{U}_{\mathrm{cal}}$ value increases by three times more than that of the optimized geometry.

To this end, here we have explored the possibility of finetuning the barrier height for magnetization reversal using oriented external electric fields in Ln(III) SIMs. Enhancement in $\mathrm{U}_{\text {cal }}$ value twice that of the X-ray structures offers a viable nonchemical fine-tuning way to enhance the barrier height beyond the limit set by the ligand fields. This novel approach expected to trigger substantial interests to obtain new generation SIMs unveiling its potential applications.

\section{ASSOCIATED CONTENT}

Supporting Information. "This material is available free of charge via the Internet at http://pubs.acs.org."

\section{AUTHOR INFORMATION}

\section{Corresponding Author}

* Gopalan Rajaraman

\section{Present Addresses}

$\dagger$ Department of Chemistry, Indian Institute of Technology Bombay, Mumbai, Maharashtra- 400076, India.

\section{ACKNOWLEDGEMENT}

AS thanks CSIR for senior research fellowship (SRF) and GR would like to thank DST/SERB (CRG/2018/000430; SB/SJF/201920/12DST/SJF/CSA-03/2018-10) for funding.

\section{REFERENCES}

1. (a) Leuenberger, M. N.; Loss, D., Quantum computing in molecular magnets. Nature 2001, 410 (6830), 789-793; (b) Sanvito, S., Molecular spintronics. Chem. Soc. Rev. 2011, 40 (6), 3336-55; (c) Moreno-Pineda, E.; Godfrin, C.; Balestro, F.; Wernsdorfer, W.; Ruben, M., Molecular spin qudits for quantum algorithms. Chem. Soc. Rev. 2018, 47 (2), 501-513.

$2 . \quad$ (a) Ding, Y. S.; Chilton, N. F.; Winpenny, R. E.; Zheng, Y. Z., On approaching the limit of molecular magnetic anisotropy: a nearperfect pentagonal bipyramidal dysprosium (III) single-molecule magnet. Angew.Chem. Int. Ed. 2016, 55 (52), 16071-16074; (b) Gupta, S. K.; Rajeshkumar, T.; Rajaraman, G.; Murugavel, R., An air-stable Dy(iii) single-ion magnet with high anisotropy barrier and blocking temperature. Chem. Sci. 2016, 7 (8), 5181-5191; (c) Liu, J.; Chen, Y.C.; Liu, J.-L.; Vieru, V.; Ungur, L.; Jia, J.-H.; Chibotaru, L. F.; Lan, Y.; Wernsdorfer, W.; Gao, S., A stable pentagonal bipyramidal Dy (III) single-ion magnet with a record magnetization reversal barrier over 1000 K. J. Am. Chem. Soc. 2016, 138 (16), 5441-5450.

$3 . \quad$ (a) Goodwin, C. A. P.; Ortu, F.; Reta, D.; Chilton, N. F.; Mills, D. P., Molecular magnetic hysteresis at 60 kelvin in dysprosocenium. Nature 2017, 548 (7668), 439-442; (b) Guo, F.-S.; Day, B. M.; Chen, Y.-C.; Tong, M.-L.; Mansikkamäki, A.; Layfield, R. A., Magnetic hysteresis up to 80 kelvin in a dysprosium metallocene single-molecule magnet. Science 2018, 362 (6421), 1400-1403; (c) Guo, F. S.; Day, B. M.; Chen, Y. C.; Tong, M. L.; Mansikkamäki, A.; Layfield, R. A., A Dysprosium Metallocene Single-Molecule Magnet Functioning at the Axial Limit. Angew.Chem. Int. Ed. 2017, 56 (38), 11445-11449; (d) McClain, K. R.; Gould, C. A.; Chakarawet, K.; Teat, S. J.; Groshens, T. J.; Long, J. R.; Harvey, B. G., High-temperature magnetic blocking and magneto-structural correlations in a series of dysprosium (III) metallocenium single-molecule magnets. Chem. Sci. 2018, 9 (45), 8492-8503; (e) Evans, P.; Reta, D.; Whitehead, G. F.; Chilton, N. F.; Mills, D. P., Bis-Monophospholyl Dysprosium Cation Showing Magnetic Hysteresis at 48 K. J. Am. Chem. Soc. 2019, 141 (50), 19935-19940; (f) Gould, C. A.; McClain, K. R.; Yu, J. M.; Groshens, T. J.; Furche, F.; Harvey, B. G.; Long, J. R., Synthesis and magnetism of neutral, linear metallocene complexes of terbium (II) and dysprosium (II). J. Am. Chem. Soc. 2019, 141 (33), 12967-12973.

4. $\quad$ Rinehart, J. D.; Fang, M.; Evans, W. J.; Long, J. R., Strong exchange and magnetic blocking in $\mathrm{N}(2)(3)(-)$-radical-bridged lanthanide complexes. Nat. Chem. 2011, 3 (7), 538-42.

5. $\quad$ Zheng, Y. Z.; Ding, Y. S.; Han, T.; Zhai, Y. Q.; Reta, D.; Chilton, N. F.; Winpenny, R. E. P., A Study of Magnetic Relaxation in Dysprosium(III) Single-Molecule Magnets. Chem. Eur. J. 2020.

6. Woodruff, D. N.; Winpenny, R. E.; Layfield, R. A., Lanthanide single-molecule magnets. Chem. Rev. 2013, 113 (7), 511048.

7. Canaj, A. B.; Dey, S.; Martí, E. R.; Wilson, C.; Rajaraman, G.; Murrie, M., Insight into D6h Symmetry: Targeting Strong Axiality in Stable Dysprosium (III) Hexagonal Bipyramidal Single-Ion Magnets. Angew. Chem. 2019, 131 (40), 14284-14289.

8. Upadhyay, A.; Singh, S. K.; Das, C.; Mondol, R.; Langley, S. K.; Murray, K. S.; Rajaraman, G.; Shanmugam, M., Enhancing the effective energy barrier of a Dy (iii) SMM using a bridged diamagnetic Zn (ii) ion. Chem. Commun. 2014, 50 (64), 8838-8841.

9. Singh, M. K.; Yadav, N.; Rajaraman, G., Record high magnetic exchange and magnetization blockade in Ln $2 @ \mathrm{C} 79 \mathrm{~N}(\mathrm{Ln}=$ Gd (III) and Dy (III)) molecules: a theoretical perspective. Chem. Commun. 2015, 51 (100), 17732-17735.

10. (a) Zyazin, A. S.; van den Berg, J. W.; Osorio, E. A.; van der Zant, H. S.; Konstantinidis, N. P.; Leijnse, M.; Wegewijs, M. R.; May, F.; Hofstetter, W.; Danieli, C.; Cornia, A., Electric field controlled magnetic anisotropy in a single molecule. Nano Lett. 2010, 10 (9), 3307-11; (b) Goswami, T.; Misra, A., On the control of magnetic anisotropy through an external electric field. Chem. Eur. J. 2014, 20 (43), 13951-6; (c) Palii, A.; Aldoshin, S.; Tsukerblat, B., MixedValence Triferrocenium Complex with Electric Field Controllable Superexchange as a Molecular Implementation of Triple Quantum Dot. J. Phys. Chem. C 2017, 121 (48), 27218-27224; (d) Fittipaldi, M.; Cini, A.; Annino, G.; Vindigni, A.; Caneschi, A.; Sessoli, R., Electric field modulation of magnetic exchange in molecular helices. Nat. Mater. 2019, 18 (4), 329-334; (e) Liu, J.; Mrozek, J.; Myers, W. K.; Timco, G. A.; Winpenny, R. E. P.; Kintzel, B.; Plass, W.; Ardavan, A., Electric Field Control of Spins in Molecular Magnets. Phys. Rev. Lett. 2019, 122 (3), 037202; (f) Boudalis, A. K.; Robert, J.; Turek, P., First Demonstration of Magnetoelectric Coupling in a Polynuclear Molecular Nanomagnet: Single-Crystal EPR Studies of [Fe3 O(O2 $\mathrm{CPh} 6$ (py)3 ]ClO4 py under Static Electric Fields. Chem. Eur. J. 2018, 24 (56), 14896-14900; (g) Weisheit, M.; Fähler, S.; Marty, A.; Souche, Y.; Poinsignon, C.; Givord, D., Electric field-induced modification of magnetism in thin-film ferromagnets. Science 2007, 315 (5810), 349351 .

11. Brown, A. J.; Pinkowicz, D.; Saber, M. R.; Dunbar, K. R., A Trigonal-Pyramidal Erbium(III) Single-Molecule Magnet. Angew. Chem. Int. Ed. 2015, 54 (20), 5864-8.

12. Singh, S. K.; Pandey, B.; Velmurugan, G.; Rajaraman, G., Key role of higher order symmetry and electrostatic ligand field design in the magnetic relaxation of low-coordinate Er(iii) complexes. Dalton Trans. 2017, 46 (35), 11913-11924.

13. Shaik, S.; Ramanan, R.; Danovich, D.; Mandal, D., Structure and reactivity/selectivity control by oriented-external electric fields. Chem. Soc. Rev. 2018, 47 (14), 5125-5145.

14. (a) Wang, C.; Danovich, D.; Chen, H.; Shaik, S., Oriented External Electric Fields: Tweezers and Catalysts for Reactivity in Halogen-Bond Complexes. J. Am. Chem. Soc. 2019, 141 (17), 7122 7136; (b) Huang, X.; Tang, C.; Li, J.; Chen, L.-C.; Zheng, J.; Zhang, P.; Le, J.; Li, R.; Li, X.; Liu, J., Electric field-induced selective catalysis of single-molecule reaction. Sci. Adv. 2019, 5 (6), eaaw3072; (c) Zakharov, A. B.; Ivanov, V. V.; Adamowicz, L., Electronic perturbation effects in the presence of electric field for $\pi$-conjugated 
systems: An electron-correlation study. Int. J. Quantum Chem., e26260.

15. Singh, S. K.; Gupta, T.; Shanmugam, M.; Rajaraman, G., Unprecedented magnetic relaxation via the fourth excited state in low- coordinate lanthanide single-ion magnets: a theoretical perspective. Chem. Commun. 2014, 50 (98), 15513-6.

16. Chandrasekhar, V.; Pointillart, F., Organometallic Magnets.

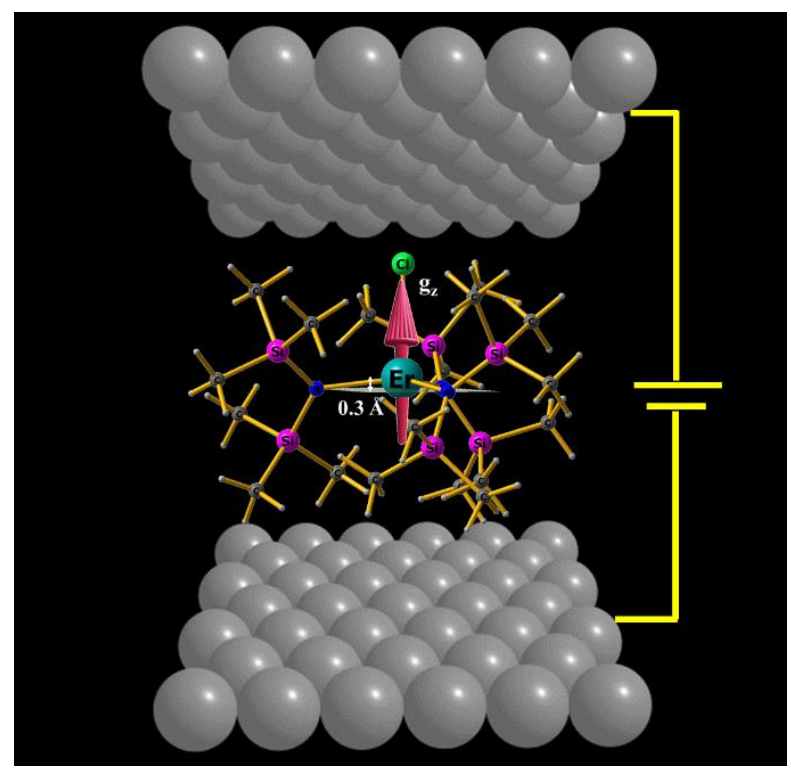

